\title{
Person-Specific Non-shared Environmental Influences in Intra- individual Variability: A Preliminary Case of Daily School Feelings in Monozygotic Twins
}

\author{
Yao Zheng ${ }^{1,2} \cdot$ Peter C. M. Molenaar ${ }^{3} \cdot$ Rosalind Arden $^{4} \cdot$ Kathryn Asbury $^{5}$. \\ David M. Almeida ${ }^{3}$
}

Received: 5 June 2015/Accepted: 25 March 2016/Published online: 4 April 2016

(c) The Author(s) 2016. This article is published with open access at Springerlink.com

\begin{abstract}
Most behavioural genetic studies focus on genetic and environmental influences on inter-individual phenotypic differences at the population level. The growing collection of intensive longitudinal data in social and behavioural science offers a unique opportunity to examine genetic and environmental influences on intra-individual phenotypic variability at the individual level. The current study introduces a novel idiographic approach and one novel method to investigate genetic and environmental influences on intra-individual variability by a simple empirical demonstration. Person-specific non-shared environmental influences on intra-individual variability of daily school feelings were estimated using time series data from twenty-one pairs of monozygotic twins (age $=10$ years, 16 female pairs) over two consecutive weeks. Results showed substantial inter-individual heterogeneity in person-specific non-shared environmental influences. The current study represents a first step in investigating environmental influences on intra-individual variability with an idiographic approach, and provides implications for future
\end{abstract}

Edited by Gitta Lubke.

Yao Zheng

yza296@sfu.ca

1 Department of Psychology, Simon Fraser University, RCB 5246, 8888 University Drive, Burnaby, BC V5A 1S6, Canada

2 Child and Family Research Institute, Vancouver, BC, Canada

3 Human Development and Family Studies, The Pennsylvania State University, University Park, PA, USA

4 Centre for Philosophy of Natural and Social Science, London School of Economics, London, UK

5 Department of Education, Psychology in Education Research Centre, University of York, York, UK behavioural genetic studies to examine developmental processes from a microscopic angle.

Keywords Intra-individual variability · Non-shared environmental influences · Daily diary data $\cdot$ Personspecific · Idiographic approach - Developmental processes

\section{Introduction}

Social and behavioural science researchers are increasingly collecting intensive longitudinal data (ILD) on various physiological, psychological, and behavioural phenotypes using study designs such as ecological momentary assessments and daily diary design (Walls and Schafer 2006). As opposed to conventional longitudinal designs that typically follow thousands or hundreds of participants a few times over multiple years, ILD are typically obtained intensively and repeatedly over a short time period (e.g., days, weeks) in a relatively smaller sample of participants (e.g., hundreds). Recently, behavioural genetic researchers are also starting to collect ILD in twin samples (e.g., Asbury et al. 2008; Kessler et al. 2004; Klump et al. 2013; Wichers et al. 2007).

ILD offer a unique opportunity to investigate dynamic developmental processes at a more refined microscopic level (e.g., days, vs. years) on phenotypes assessed in realtime (e.g., momentary ambulatory measures, vs. recall past year behaviours in questionnaires) and ecological or daily life contexts (e.g., office, vs. laboratory) (Shiffman et al. 2008; Stone et al. 2007). Particularly, ILD enable the examination of intra-individual variability, which has been shown to contain important and unique information on inter-individual differences for a variety of phenotypes (Diehl et al. 2015; Nesselroade 1991; Nesselroade and Ram 2004; Ram and Gerstorf 2009). As opposed to intra- 
individual change measured in conventional longitudinal studies that typically involves systematic and irreversible mean changes (e.g., growth of height and intelligence), intra-individual variability typically involves relatively short-term fluctuations that are generally reversible without substantial mean changes (e.g., daily mood fluctuation) (Nesselroade 1991; Nesselroade and Ram 2004; Ram and Gerstorf 2009).

Intra-individual variability enables us to examine the dynamic intra-individual developmental pattern at the individual level through single-subject analysis such as dynamic factor analysis (Molenaar 1985). Additionally, ILD obtained in multiple individuals enable us to examine inter-individual differences in intra-individual developmental patterns (Nesselroade 1991; Nesselroade and Ram 2004; Ram and Gerstorf 2009). This presents some novel and open questions to behavioural genetic research: What are the genetic and environmental influences on intra-individual variability at the individual level? How do individuals differ from each other regarding their respective genetic and environmental influences on intra-individual variability? How do results of genetic and environmental influences on intra-individual variability compare to results of genetic and environmental influences on inter-individual differences, or inter-individual differences in intra-individual changes that typically are examined in conventional behavioural genetic studies? The few existing empirical studies that have collected genetically informative ILD did not specifically examined genetic and environmental influences on intra-individual variability (e.g., Asbury et al. 2008; Burt et al. 2015; Cleveland and Almeida 2013; Jacobs et al. 2006, 2013; Menne-Lothmann et al. 2012; Neiss and Almeida 2004; Wichers et al. 2007), and thus could not shed light on these questions.

Classic ergodic theory (e.g., Birkhoff 1931) provides some insight to the question on the relation between results of genetic and environmental influences on intra-individual variability and results of genetic and environmental influences on inter-individual differences. The classic ergodic theory is a general mathematical theory that describes the relations between analyses of inter-individual variation (i.e., inter-individual differences) and analyses of intra-individual variation (i.e., intra-individual variability). According to the classical ergodic theorems, for Gaussian processes, results obtained from analyses of inter-individual variation and of intra-individual variation would be equivalent to each other only when two major assumptions are met: the population homogeneity assumption and the stationarity assumption (Molenaar 2004; Molenaar and Campbell 2009). Inter-individual analyses can show satisfactory model fit while being completely insensitive to intra-individual variation (Kelderman and Molenaar 2007). Relating to behavioural genetic research, the population homogeneity assumption implies that genetic and environmental influences are the same for all individuals in the population, while the stationarity assumption states that both the mean levels and sequential (co)variances of phenotypes remain constant over time. As theoretically elaborated and empirically shown, however, these two assumptions are typically violated (see Molenaar 2004, 2007, 2010a, 2014). For example, behavioural genetic studies adopting mixture modelling have demonstrated population heterogeneity regarding genetic and environmental influences (e.g., Eaves et al. 1993; Gillespie and Neale 2006; Muthén et al. 2006; Neale 2014). Stochastic intra-individual developmental noise (e.g., Molenaar et al. 1993; Molenaar and Raijmakers 1999), gene expression and epigenetic processes (e.g., DNA methylation) can also result in substantial inter-individual differences in genetic and environmental influences (e.g., Bell and Saffery 2012; Bell and Spector 2011; Dolan et al. 2015; Fraga et al. 2005; Kan et al. 2010; Petronis 2010; Wright et al. 2014). Therefore, investigation of intra-individual variability could potentially provide unique and important information regarding genetic and environmental influences on intra-individual developmental processes that conventional behavioural genetic studies focusing on inter-individual differences could not offer, especially at the individual level.

Some progress of behavioural genetic research on intraindividual variability has been made that could provide potential opportunity for researchers to investigate genetic and environmental influences at the individual level, and to uncover inter-individual heterogeneity in genetic and environmental influences. Nesselroade et al. (2007) recently introduced the concept of idiographic filter (IF) in measurement, which allows person-specific factor loadings and residual variances, while defining measurement equivalence at the factor level by constraining their intercorrelations to be invariant across individuals. A hybridized model was later proposed that combines the IF and biometric model together, acronized as iFACE model, to model person-specific genetic and environmental influences on intra-individual variability within twin pairs (Molenaar 2010a, 2014; Molenaar et al. 2012; Nesselroade and Molenaar 2010). Upon its recent development, iFACE model has been applied to single pairs of dizygotic (DZ) twins on multivariate electroencephalogram (EEG) recordings data. Results showed considerable personspecific genetic and environmental influences within the same twin pair, demonstrating the violation of population homogeneity assumption (Molenaar et al. 2012). The primary goal of the current study is to further introduce the iFACE model and to demonstrate its utility and feasibility by applying the iFACE model to daily mood data from a genetically informative short daily diary study with monozygotic (MZ) twins. 
Intra-individual variability of mood has been widely studied. People's moods fluctuate over time in substantial and meaningful ways (Eid and Diener 1999). Research has shown that factor patterns explaining intra-individual variability differ from factor patterns explaining inter-individual differences (e.g., Watson 1988; Zevon and Tellegen 1982), and has revealed substantial inter-individual heterogeneity in dynamic intra-individual patterns of mood (e.g., Ferrer and Nesselroade 2003). Some people's moods fluctuate quickly; others' moods change more slowly. These inter-individual differences in intra-individual patterns of mood can be predicted by individual-level characteristics (e.g., Chow et al. 2005). In addition, previous studies have found associations between daily mood and daily social interactions and experience (e.g., Clark and Watson 1988). These findings suggest that intra-individual processes of mood are subject to significant environmental influences. Therefore, when examining phenotypes like mood, it is also important to examine intra-individual variability at the individual level, as well as inter-individual differences in these intra-individual patterns.

Twin studies have examined genetic and environmental influences on inter-individual differences in positive and negative mood, and generally found them to be affected by genetic and non-shared environmental influences, although the estimates vary depending on different sample sizes, age ranges, and specific measures (e.g., Baker et al. 1992; Gatz et al. 1992; Riemann et al. 1998). As mentioned earlier, the few existing empirical studies that have collected genetically informative ILD on mood did not specifically examined genetic and environmental influences on intra-individual variability, but focused instead on inter-individual differences in constructs aggregated together over multiple observations, such as intra-individual mean and variation (e.g., Jacobs et al. 2006, 2013; Menne-Lothmann et al. 2012; Neiss and Almeida 2004; Wichers et al. 2007). Among those studies, however, one study that followed 239 female twin pairs aged 16-25 years over a 7-day period provided some interesting results that could shed some lights on genetic and environmental influences on intra-individual variability of affect (Burt et al. 2015). Besides the finding that both positive and negative affect were primarily explained by genetic (25-47\%) and non-shared environmental influences (54-75\%), it was also found that genetic stability remained high across days $(\sim .95)$, whereas the stability of non-shared environmental influences was low and decreased monotonically with increasing time intervals (Burt et al. 2015). This finding suggests that non-shared environmental influences could consist primarily of idiosyncratic and transient daily experience, and highlights the importance of examining intra-individual processes, particularly with regard to mood.

Using data from a genetically informative short daily diary study with MZ twins, the current study aimed to demonstrate the utility of iFACE model by examining person-specific non-shared environmental influences on intra-individual variability of daily mood. In intra-individual behavioural genetic analysis of intra-individual variability, the time series data of a single MZ or DZ twin pair are considered. As the additive genetic factor is indistinguishable from the shared environmental factor among MZ twins, the current study focused on familiality (additive genetic and shared environmental influences) versus non-shared environmental influences (see Molenaar et al. 2012 for an application in DZ twin pairs). We are particularly interested to know how individuals differ from each other regarding their person-specific non-shared environmental influences.

\section{Methods}

\section{Sample and data collection procedure}

The analysis sample consists of twenty-one MZ twin pairs drawn from the 1994/1995 cohort of the Twins Early Development Study (TEDS). TEDS is a longitudinal study of twins born in England and Wales between 1994 and 1996; the twins have been followed since infancy. All pairs were 10-year-olds and in the penultimate year of elementary schooling in the United Kingdom. Both twins in each pair were pupils in the same classroom. The sample mean SES (-.02) is close to the mean for the full TEDS sample (0.00), which has been shown to be representative of the UK population (Haworth et al. 2013). The majority of the twin pairs were female (16 pairs) and White (19 pairs). Trained interviewers conducted daily telephone interviews during the same two consecutive weeks every weekday evening after school. Each interview lasted 5-10 min per day for each child. Therefore, each twin provided up to 10 days of diary data, and a total of 402 observations were collected. Details of sample recruitment and interview procedure are described elsewhere (Asbury et al. 2008). It is noteworthy that the original sample includes a total of sixty-one $\mathrm{MZ}$ twin pairs. However, many twin pairs showed little or no intra-individual variability in their daily school feelings in either one or both twins in each pair during the short sampling period. Therefore, they were excluded from further analysis.

\section{Measures}

\section{Daily positive school feelings}

This construct was measured by combining two sub-scales, one measuring daily positive feelings about classroom experiences and the other about playground experiences. 
For each sub-scale, four items were used with possible responses ranging from 1 "very" to 4 "not at all". An average score was calculated from the combined subscales. An example item asked: did you feel proud in the classroom today? Other adjectives included interested, excited, and enthusiastic. Items were reverse coded so that higher scores indicated more positive school feelings (ranging from 1 to 4 ).

\section{Daily negative school feelings}

This construct was measured by combining two sub-scales, one measuring daily negative feelings about classroom experiences and the other about playground experiences. For each sub-scale, four items were used with possible responses ranging from 1 "very" to 4 "not at all". An average score was calculated from the combined sub-scales. An example item asked: did you feel nervous in the playground today? Other adjectives included upset, scared, and irritable. Items were reverse coded so that higher scores indicated more negative school feelings (ranging from 1 to 4 ).

\section{Analytic strategy}

We tested the data against the idiographic filter ACE (iFACE) model that combines the idiographic filter (IF) with a conventional biometric model to decompose intra-individual phenotypic variability into three independent factors, additive genetics (A), shared environment (C), and nonshared environment (E) (Molenaar et al. 2012; Nesselroade and Molenaar 2010). The iFACE model is analogous to the standard longitudinal genetic factor model that decomposes inter-individual phenotypic variation in the population (Boomsma and Molenaar 1987; Martin and Eaves 1977; Molenaar and Boomsma 1987). The identification and interpretation of the genetic and environmental factors are obtained by their inter-correlation patterns within the pair. A major difference between the iFACE model and a longitudinal genetic factor model is that, while the latter constrains factor loadings and residuals to be invariant both between twin pairs and between members in the same twin pair, the iFACE model allows for person-specific factor loadings and residuals as they are estimated from individual time series data. This enables the estimation of person-specific genetic and environmental influences. Applying an iFACE model to time series data for each member of a twin pair does not invoke the population homogeneity assumption; it can also uncover person-specific heterogeneity in intra-individual genetic and environmental factor loadings.

Because MZ twins share all of their genes, their latent additive genetic factor is indistinguishable from the latent shared environmental factor, which is the same within each twin pair. Therefore, the current analysis can only distinguish two latent factors: the non-shared environment (E), as well as the additive genetic and shared environment factor combined together (AC, or familiality). As shown in Fig. 1, each twin's positive and negative school feelings measured at one time $t$ (e.g., $\mathrm{NSF}_{1, \mathrm{t}}, \mathrm{PSF}_{1, \mathrm{t}}$ ) have factor loadings on two latent factors, $\mathrm{AC}_{1, \mathrm{t}}$ and $\mathrm{E}_{1, \mathrm{t}}$ (e.g., $\alpha_{1,2}$ for the AC path on $\mathrm{PSF}_{1, \mathrm{t}}$ ). Each phenotype also has its residual (e.g., $\mathrm{e}_{1,1}$ for $\mathrm{NSF}_{1, t}$ ) which could contain specific genetic and environmental influences. The same specification applies to the same phenotypes measured at the next time $\mathrm{t}+1$ (e.g., $\mathrm{NSF}_{1, \mathrm{t}+1}, \mathrm{PSF}_{1, \mathrm{t}+1}$ ), and the respective factor loadings are constrained to be the same (e.g., $\phi_{1,1}$ for the $\mathrm{E}$ path of $\mathrm{NSF}_{1, \mathrm{t}}$ and $\mathrm{NSF}_{1, \mathrm{t}+1}$ ). Therefore, genetic and environmental influences estimated in each individual iFACE model are average effects across each person's measured time. A first-order autoregression (factors at time $\mathrm{t}+1$ predicted by factors at the previous time $\mathrm{t}$ ) is used to model the stability of the latent $\mathrm{AC}$ and $\mathrm{E}$ factors with person-specific autoregressive coefficient for $\mathrm{E}\left(\gamma_{1}\right)$. The same specification applies to the co-twin's time series data, however, with person-specific factor loadings (e.g., $\alpha_{2,2}$ for the AC path on $\mathrm{PSF}_{2, t}$ ) and residuals (e.g., $\mathrm{e}_{2,1}$ for $\mathrm{NSF}_{2, t}$ ), giving person-specific estimates of genetic and environmental influences. The prediction residual for $\mathrm{E}(\chi)$ was uncorrelated in the twin pair by definition, and correlated at 1 in the twin pair for $\mathrm{AC}(\xi)$, leading the prediction coefficient $(\beta)$, or the stability of $A C$, to be the same for both twins in the same pair. The phenotypic residuals (e) and prediction residuals $(\chi$ and $\xi$ ) for each twin are assumed to be multinormally distributed within each individual time series data. A more detailed explanation for iFACE model in the general multivariate case with mathematic formula and model identification proof is provided elsewhere (Molenaar et al. 2012).

All iFACE models were fit using the Fortran program MKFM6 (Dolan 2005) that implements Harvey's time-invariant Kalman filter algorithm (Harvey 1989) to calculate normal theory maximum likelihood estimates of multivariate stationary time series model (The MKFM6 program is freely available from http://quantdev.ssri.psu.edu/resour ces). This algorithm seeks to minimize the difference, or prediction error, between the predicted value based on all previous observations $E\left[\mathrm{y}_{\mathrm{t}} \mid \mathrm{Y}_{\mathrm{t}-1}\right]$, where $\mathrm{Y}_{\mathrm{t}-1}=\left\{\mathrm{y}_{1}\right.$, $\left.\mathrm{y}_{2}, \ldots, \mathrm{y}_{\mathrm{t}-1}\right\}$, and the actual observation value $\mathrm{y}_{\mathrm{t}}$. A quasiNewton optimization routine using exact gradients is used to maximize the loglikelihood function; missing values during weekend days were coded as missing and handled by the MKFM6 algorithm (Dolan 2005). The iFACE model can also be equivalently represented in Toeplitz format summarizing time lag covariance in block Toeplitz matrix and fit in any structural equation modelling software (e.g., Molenaar et al. 2012).

The iFACE model was first fitted to individually standardized time series data. As a reference for comparison, a group-level model with the same model specification as in the 
Fig. 1 Path diagram of iFACE model in one monozygotic twin pair. NSF, negative school feelings, $P S F$ positive school feelings, $A C$ additive genetic and shared environmental factor, E, non-shared environmental factor

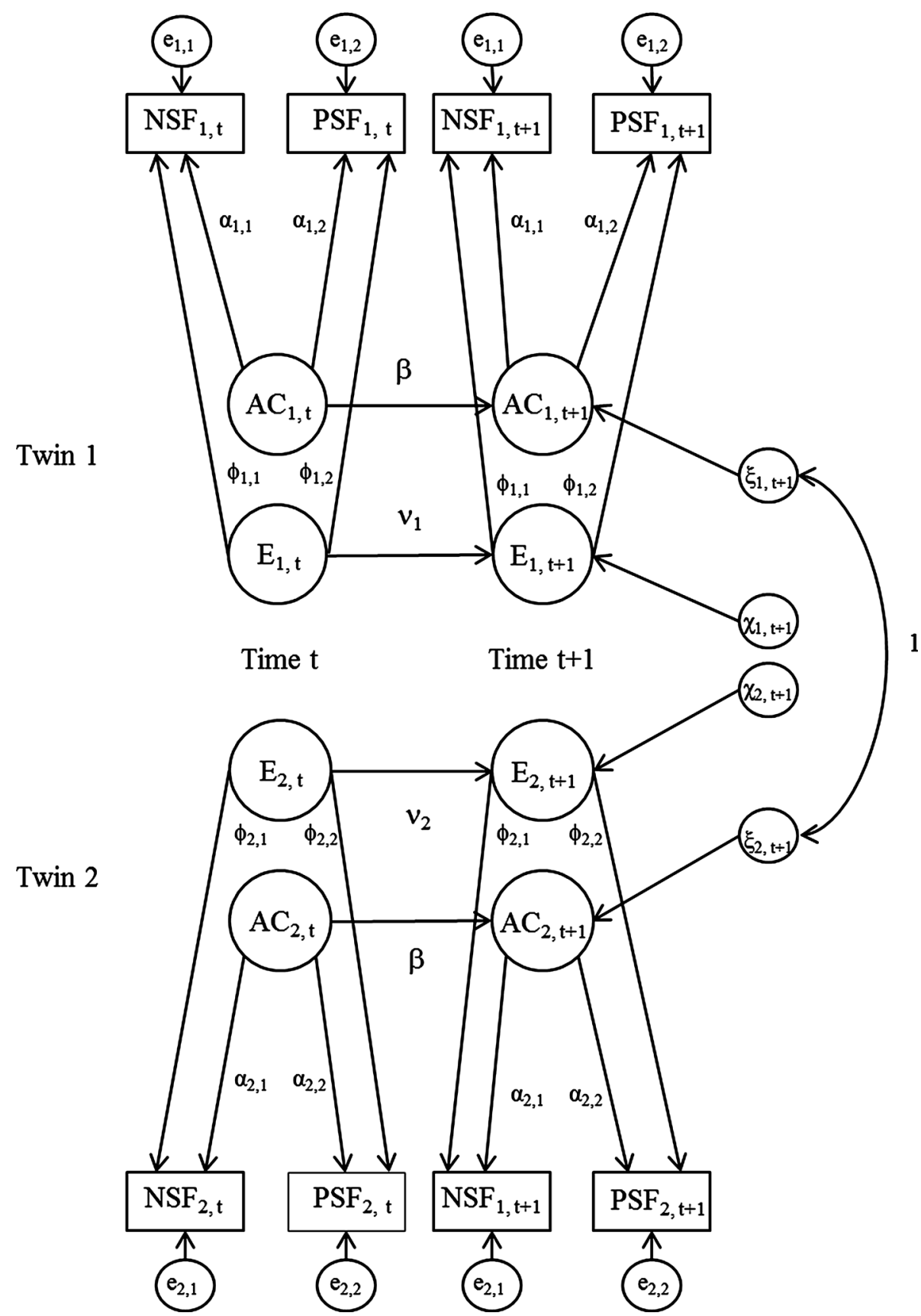

individual iFACE model but constraining all parameters (e.g., factor loadings, residuals) to be the same across all individuals was also performed, therefore assuming genetic and environmental influences to be the same for all individuals.

\section{Results}

\section{Descriptive statistics}

As shown in Table 1, the group grand mean (averaged over all individuals and days) of positive school feelings was
$2.80(S D=0.62)$, with individual means ranging between 1.66 and 3.90 out of the potential $1-4$ range. The group mean of negative school feelings was $1.29(S D=0.35)$, with individual means ranging between 1.03 and 2.00. Therefore, in general, pupils reported "quite" positive and "not very" negative about school every day.

\section{Group-level and individual-level non-shared environmental influences}

Group-level results showed that non-shared environmental influences accounted for $73 \%$ of the intra-individual 
variability in positive school feelings (see Table 2). Children varied in the magnitude of their person-specific nonshared environmental influences ranging from 0 to almost 1. The average of person-specific non-shared environmental influences was $0.52(S D=0.32$, skewness $=-.21$, kurtosis $=-1.27$; see Fig. 2). Using $60 \%$ as a subjective cut-off for the estimated magnitudes of person-specific non-shared environmental influences, the twin concordance rate regarding person-specific non-shared environmental influences (non-shared environmental influences of both twins in the same pair were lower or higher than $60 \%$ ) was $48 \%$. Seven concordant pairs showed the same pattern as in the group-level results in that their daily positive school feelings were primarily accounted for by non-shared environmental influences, ranging from approximately $60 \%$ to almost $100 \%$ (e.g., pair 14). Three concordant pairs showed the opposite pattern-daily positive school feelings of both twins primarily explained by familiality, with non-shared environmental influences ranging from almost 0 to $25 \%$ (e.g., pair 12).

The remaining eleven discordant pairs demonstrated two patterns. First, among six discordant pairs, one twin's daily positive school feelings were primarily explained by nonshared environmental influences as in the group-level result, whereas the co-twin's was mostly explained by familiality (e.g., 3 vs. $60 \%$ in pair 4 ). The remaining five pairs showed a pattern where one twin's positive feelings were either primarily explained by non-shared environmental influences (e.g., twin 2 in pair 2) or familiality (e.g., twin 1 in pair 19), whereas the co-twin's was explained about half $(\sim 50 \%)$ by non-shared environmental influences.

Group-level results showed that the intra-individual variability of negative school feelings was largely explained by familiality, with only a small portion attributed to non-shared environmental influences (16\%). Children also varied in person-specific non-shared environmental influences, with an average of $0.51(S D=0.37$, skewness $=.07$, kurtosis $=-1.62$; see Fig. 2 ). The concordance rate for negative school feelings was $43 \%$. Specifically, five concordant pairs showed the same pattern as the group-level results, with minimal non-shared environmental influences ranging from 2 to $39 \%$ (e.g., pair 1). Another four concordant pairs showed the opposite pattern in that their daily negative school feelings were mostly explained by non-shared environmental influences for both twins, ranging from $66 \%$ to almost $100 \%$ (e.g., pair 21).

The remaining twelve discordant pairs demonstrated two patterns. First, for nine of these pairs, one twin's daily negative school feelings was primarily explained by familiality as in the group-level results, whereas the cotwin's was primarily explained by non-shared environmental influences (e.g., 15 vs. $82 \%$ in pair 5). The
Table 1 Group and individual means (SDs) of daily school feelings

\begin{tabular}{|c|c|c|c|}
\hline Pair & Twin & Positive school feelings & Negative school feelings \\
\hline \multicolumn{2}{|l|}{ Group } & $2.80(.62)$ & $1.29(.35)$ \\
\hline \multirow[t]{2}{*}{1} & 1 & $2.03(.51)$ & $1.09(.12)$ \\
\hline & 2 & $3.16(.24)$ & $1.16(.14)$ \\
\hline \multirow[t]{2}{*}{2} & 1 & $3.04(.28)$ & $1.42(.31)$ \\
\hline & 2 & $3.06(.47)$ & $1.08(.11)$ \\
\hline \multirow[t]{2}{*}{3} & 1 & $3.71(.28)$ & $1.10(.16)$ \\
\hline & 2 & $3.90(.14)$ & $1.60(.32)$ \\
\hline \multirow[t]{2}{*}{4} & 1 & $3.30(.13)$ & $1.09(.10)$ \\
\hline & 2 & $2.76(.33)$ & $1.03(.05)$ \\
\hline \multirow[t]{2}{*}{5} & 1 & $2.40(.37)$ & $1.29(.19)$ \\
\hline & 2 & $2.10(.46)$ & $1.13(.17)$ \\
\hline \multirow[t]{2}{*}{6} & 1 & $2.49(.68)$ & $1.64(.38)$ \\
\hline & 2 & $3.28(.33)$ & $1.08(.12)$ \\
\hline \multirow[t]{2}{*}{7} & 1 & $2.53(.31)$ & $1.25(.23)$ \\
\hline & 2 & $2.61(.36)$ & $1.10(.12)$ \\
\hline \multirow[t]{2}{*}{8} & 1 & $2.73(.23)$ & $1.33(.17)$ \\
\hline & 2 & $3.09(.12)$ & $1.14(.27)$ \\
\hline \multirow[t]{2}{*}{9} & 1 & $2.33(.30)$ & $1.23(.23)$ \\
\hline & 2 & $2.83(.19)$ & $1.18(.18)$ \\
\hline \multirow[t]{2}{*}{10} & 1 & $3.16(.30)$ & $1.23(.19)$ \\
\hline & 2 & $2.83(.42)$ & $1.36(.31)$ \\
\hline \multirow[t]{2}{*}{11} & 1 & $2.26(.29)$ & $1.31(.20)$ \\
\hline & 2 & $2.69(.35)$ & $1.70(.23)$ \\
\hline \multirow[t]{2}{*}{12} & 1 & $2.97(.30)$ & $1.11(.12)$ \\
\hline & 2 & $3.48(.48)$ & $1.49(.37)$ \\
\hline \multirow[t]{2}{*}{13} & 1 & $3.36(.48)$ & $1.13(.13)$ \\
\hline & 2 & $3.57(.46)$ & $1.10(.25)$ \\
\hline \multirow[t]{2}{*}{14} & 1 & $3.03(.29)$ & $1.30(.24)$ \\
\hline & 2 & $2.72(.22)$ & $1.05(.11)$ \\
\hline \multirow[t]{2}{*}{15} & 1 & $3.14(.11)$ & $1.15(.13)$ \\
\hline & 2 & $2.79(.31)$ & $1.60(.33)$ \\
\hline \multirow[t]{2}{*}{16} & 1 & $2.48(.30)$ & $1.89(.19)$ \\
\hline & 2 & $2.69(.12)$ & $1.55(.13)$ \\
\hline \multirow[t]{2}{*}{17} & 1 & $1.91(.42)$ & $1.43(.35)$ \\
\hline & 2 & $2.43(.77)$ & $2.00(.88)$ \\
\hline \multirow[t]{2}{*}{18} & 1 & $3.09(.30)$ & $1.08(.13)$ \\
\hline & 2 & $3.09(.53)$ & $1.09(.16)$ \\
\hline \multirow[t]{2}{*}{19} & 1 & $1.66(.50)$ & $1.15(.20)$ \\
\hline & 2 & $2.03(.37)$ & $1.14(.28)$ \\
\hline \multirow[t]{2}{*}{20} & 1 & $2.25(.59)$ & $1.14(.14)$ \\
\hline & 2 & $2.54(.79)$ & $1.45(.50)$ \\
\hline \multirow[t]{2}{*}{21} & 1 & $3.18(.31)$ & $1.33(.40)$ \\
\hline & 2 & $3.23(.40)$ & $1.34(.48)$ \\
\hline
\end{tabular}

remaining 3 pairs showed a pattern in which one twin's negative school feelings were either primarily explained by familiality (e.g., twin 1 in pair 9) or non-shared environmental influences (e.g., twin 2 in pair 7), whereas the co- 


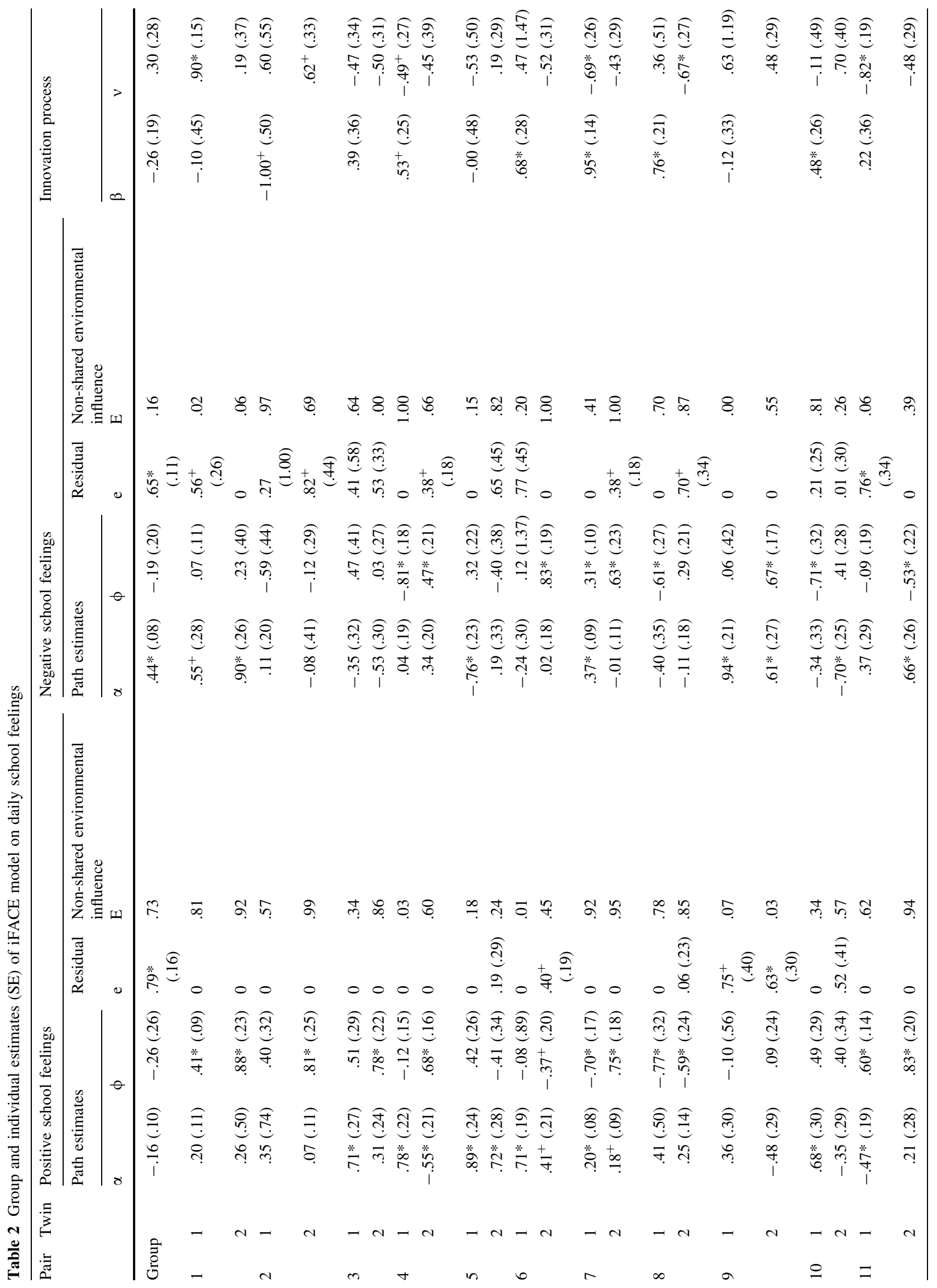




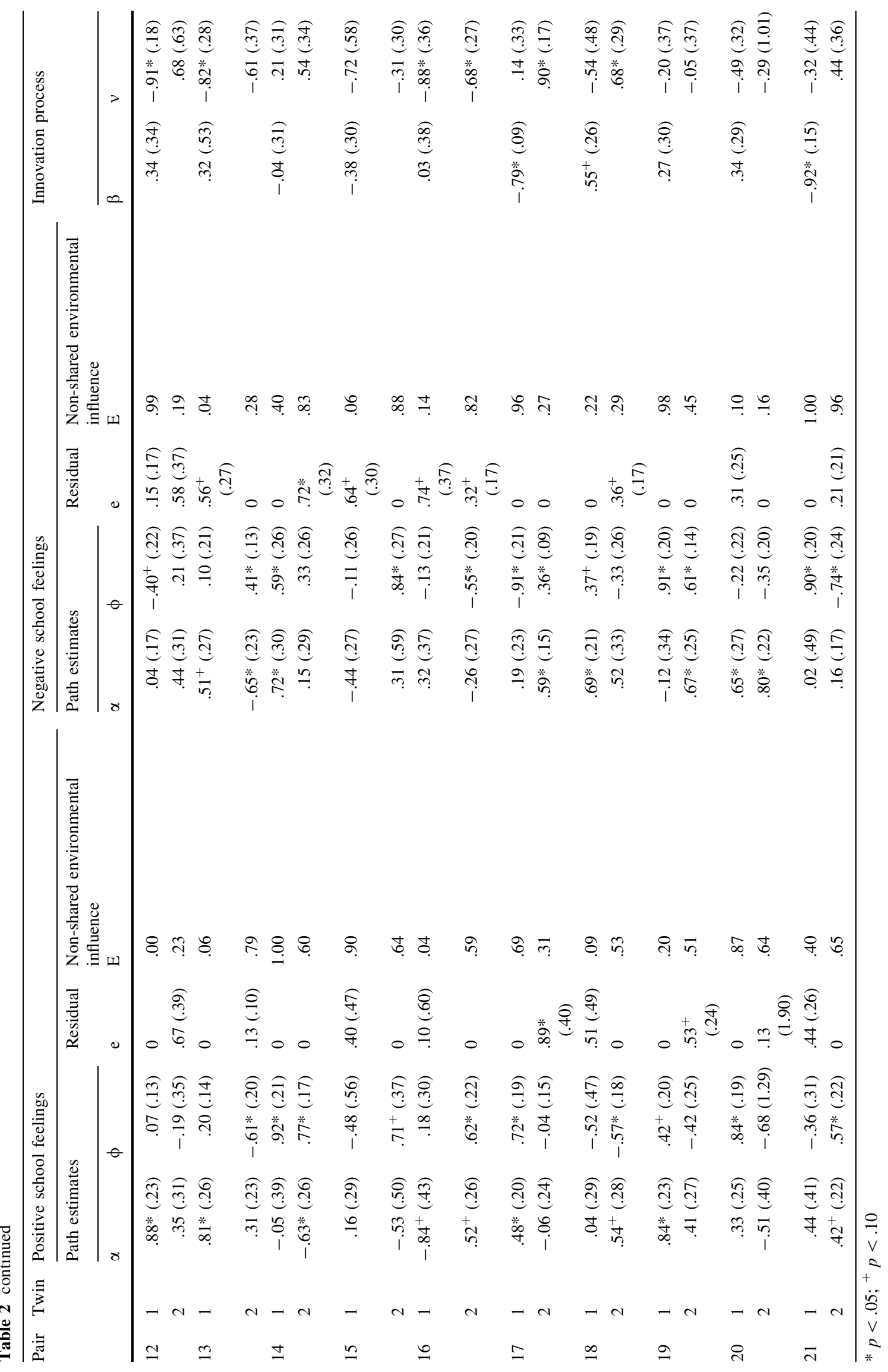



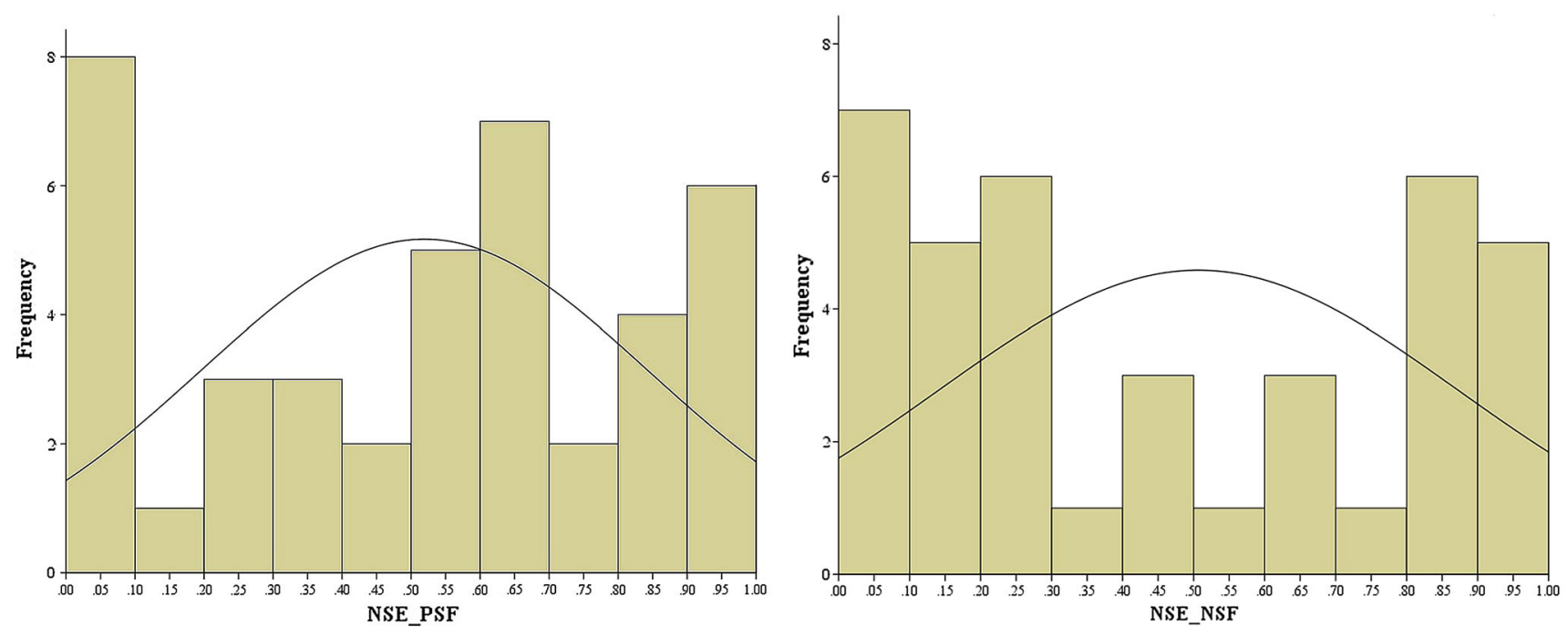

Fig. 2 Histograms of individual estimates of non-shared environmental influences for positive school feelings (left) and negative school feelings (right). NSE non-shared environmental influences. PSF positive school feelings, NSF negative school feelings

twin's was approximately half explained by non-shared environmental influences.

The group-level results showed a stability of .30 over 1 day lag for non-shared environmental factor. Therefore, about $9 \%$ of the variance of non-shared environmental factor overlapped with the previous day's non-shared environmental factor. However, individual estimates of this stability coefficient range from -0.91 to 0.90 , with a mean of $-.09(S D=.56)$.

For positive and negative school feelings respectively, individual estimates of non-shared environmental influences were not significantly correlated with individual time series mean scores $(r=.06$ and $-.20, n s)$, individual variance $(r=-.10$ and $-.08, n s)$, or individual standardized and squared time series mean scores $(r=-.05$ and $-.18, n s)$. Lastly, the group-level model that fully constrained all parameters to be the same across individuals had lower fit indices $(-2 \log$ liklihood $=1072.54$, $\mathrm{AIC}=1088.54$, BIC $=1120.59$ ) than the fully unconstrained model that specified all parameters to be personspecific $\quad(-2 \log l i k l i h o o d=727.76, \quad$ AIC $=1265.76$, $\mathrm{BIC}=2343.47)$.

\section{Discussion}

The primary goal of the current study was to demonstrate the utility and feasibility of the iFACE model (Molenaar et al. 2012; Nesselroade and Molenaar 2010) in examining genetic and environmental influences on intra-individual variability at the individual level. By applying the model to daily mood data from a genetically informative daily diary study with a small sample of MZ twins, the results demonstrated substantial inter-individual heterogeneity regarding person-specific non-shared environmental influences. Specifically, group-level results showed substantial non-shared environmental influences for positive school feelings $(73 \%)$, but modest non-shared environmental influences for negative school feelings (16\%). The nonshared environmental factor had a low stability $(r \sim 30)$ over 1 day lag, suggesting that it changed substantially across days and may consist primarily of idiosyncratic and transient experiences (Burt et al. 2015).

The individual-level results revealed substantial interindividual heterogeneity in person-specific non-shared environmental influences in that its magnitude varies greatly across individuals. Group-level estimates could describe some but not all of the pupils' intra-individual patterns in the sample. This clear violation of the population homogeneity assumption indicates that the ergodic theorem does not hold in the current situation (Molenaar 2004; Molenaar and Campbell 2009). Therefore, investigation of genetic and environmental influences on intraindividual variability can uncover unique information on intra-individual developmental processes that conventional behavioural genetic studies could not offer, especially at the individual level. There are a few possible explanations to this inter-individual heterogeneity in person-specific non-shared environmental influences. First, stochastic intra-individual developmental noise can lead to inter-individual differences in genetic and environmental influences as a third source, which however cannot be distinguished from non-shared environmental influences in conventional behavioural genetic studies (e.g., Molenaar et al. 1993; Molenaar and Raijmakers 1999). Second, epigenetic processes (e.g., DNA methylation) involving 
interactions between genes, environment, and other processes could also affect gene expression to lead to interindividual heterogeneity (e.g., Bell and Saffery 2012; Bell and Spector 2011; Dolan et al. 2015; Fraga et al. 2005; Kan et al. 2010; Petronis 2010; Wright et al. 2014).

These person-specific non-shared environmental influences offer a unique angle to examine twin concordance, not in their phenotypes per se (e.g., levels of happiness), but in their respective individual patterns of how nonshared environment influences them. In other words, there are between-pair differences with regard to the similarity between twins of their person-specific non-shared environmental influences. The finding that individual estimates of non-shared environmental influences were not significantly correlated with conventional phenotypes (e.g., individual means, variance) suggests that they could provide unique information about development above and beyond conventional phenotypes. By viewing personspecific non-shared environmental influence as a new phenotype that contains unique information on intra-individual developmental processes (the extent to which one's daily mood is influenced by non-shared environmental experience), one is able to further examine inter-individual differences in intra-individual patterns. For example, the finding that twin pair concordance in person-specific nonshared environmental influences could be summarized by a few general patterns suggests that there could be finite homogeneous subgroups of twin pairs regarding withinpair patterns in the population. Additionally, personspecific non-shared environmental influences offer another perspective on why twins growing up in the same family can be so different (Plomin 2011; Plomin and Daniels 1987). They differ not only in their non-shared environmental experiences, but also in the degree to which their daily unique environmental experiences influence them.

The current study has a few limitations that warrant caution and consideration in future studies. First, and most important, the time series data are short. Therefore, we do not over-emphasize our substantive results, but focus more on the implications that our results offer regarding the roles of genetic and environmental influences in intra-individual developmental processes. The key point here is to show empirically that this alternative approach provides new insights that take us beyond conventional behavioural genetic studies to ask questions about particular individuals as well as genetic and environmental contributions to intraindividual processes. The few existing studies that have collected ILD in twin samples, including the one from which we drew the data for analyses (Asbury et al. 2008; Kessler et al. 2004; Klump et al. 2013; Wichers et al. 2007), were not intended to examine genetic and environmental influences on intra-individual variability and all employed designs of a short time length, typically ranging for a few days. Another issue related to the short time series data is that many children in the current sample did not demonstrate any or much intra-individual variability to be included in the analysis, an issue the few previous studies encountered as well (e.g., Jacobs et al. 2013; Menne-Lothmann et al. 2012). Additionally, as opposed to conventional multivariate behavioural genetic models where common and specific genetic and environmental loadings can be specified for each phenotype as in Cholesky decomposition (Loehlin 1996), the current iFACE model only modelled the communal part of positive and negative school feelings. The residuals of positive and negative school feelings can still contain their specific genetic and environmental influences. However, such model would require more parameters and is beyond the capability of the data. With sufficiently long time series data, however, iFACE model could be extended to examine common and specific genetic and environmental influences in multiple phenotypes. It is important to note that measurement errors could lead to biased estimates of individual non-shared environmental influences and could possibly explain part of the inter-individual heterogeneity as well. With sufficiently long time series data, measurement errors could be more efficiently dealt with by adopting a psychometric common pathway model with a latent construct loading on item-level data, whose variance represents intraindividual variability that could be further decomposed with iFACE model. Rigorous studies design collecting ILD among twin samples is beyond the scope of the current study, but we refer interested readers to further readings for more information (Diehl et al. 2015; Shiffman et al. 2008; Stone et al. 2007; Walls and Schafer 2006). Simulation studies are needed to look into the optimal sample size and length of time series data needed, as well as potential influences of various signal-to-noise ratio, to reach satisfactory power and to guide future study design.

Second, while the generalizability issue is usually emphasized, it is important to note that this alternative idiographic approach does not mean single-subject analysis (Molenaar 2010b). The idiographic approach introduced in this study starts by analysing one specific individual's intra-individual variability to extract the dynamic pattern that characterizes the individual's development. Next, the same procedure is applied to many individuals' intra-individual variability separately. Individuals with the same or similar developmental patterns are grouped together to reach subgroup-level results. This idiographic behavioural genetic approach is capable of capturing inter-individual differences in the dynamic intra-individual patterns. Notably, based upon model fit indices, the fully constrained group-level model provided a more parsimonious fit to the overall data with lower AIC and BIC. This is not surprising in some way because the fully unconstrained individual- 
level models are highly parameterized in that each twin has his/her own person-specific parameter. In other words, the relative accuracy of person-specific individual-level models describing individual time series data comes with the trade-off of estimating more parameters than the grouplevel (i.e., fixed effect) model that more parsimoniously describes group data. More advanced statistical techniques are to be developed to describe and model these personspecific estimates with fewer and more sufficient parameters. For example, mixture modelling can be incorporated in future studies to identify latent subgroups with similar genetic and environmental influences on intra-individual variability (e.g., Hunter et al. 2014). Random effect models could also be considered to relate individual-level with population-level genetic and environmental influences (Dolan et al. 2015). Recent development in dynamical systems models can also be used to examine genetic and environmental influences on dynamic system parameters that describe unique intra-individual developmental pattern (e.g., Boker et al. 2014).

For the 21 pairs included in the analyses, each individual time series data were standardized, which essentially removed the intercept in the iFACE model. Therefore, estimates for each twin time series data represented nonshared environmental influences on intra-individual variability without the potential confounding of inter-individual differences in mean levels of each twin time series data (e.g., one individual generally had higher levels of negative school feelings than another). Correspondingly, the grouplevel analysis estimated non-shared environmental influences on intra-individual variability across all 21 twin pairs without confounding of inter-individual differences in mean levels while assuming homogeneity. As noted, 40 MZ pairs were excluded from analyses because of the lack of intra-individual variability (e.g., reporting no negative school feelings at all across all 10 days, or only 1 or 2 days out of 10 days, with some levels of negative school feelings). This suggests that for these $40 \mathrm{MZ}$ pairs, their interindividual differences lie more in the mean levels of school feelings than in intra-individual variability. In this case, using the aggregated mean scores over the assessed period would be a better way (e.g., Asbury et al. 2008; Cleveland and Almeida 2013; Neiss and Almeida 2004), which would produce non-shared environmental influences as in conventional behavior genetic studies using discordant monozygotic twin control design.

Behavioural genetic studies have made a tremendous contribution to our understanding of the interplay of genetics and environment in shaping human development. These studies generally focus on inter-individual phenotypic difference, or inter-individual differences in intraindividual change, which are informative at a macroscopic level - at the population level and at the macro time scale (e.g., years). As developmental science is moving toward a more refined understanding of the dynamic developmental process accompanied by the growing collection of ILD in social and behavioural science, the current study introduces a complementary idiographic approach that focuses on intra-individual phenotypic variability. Resonant with the call for personalized treatment and medicine (Collins 2010) and personalized education (Asbury and Plomin 2013) based on each individual's specific genomics that emphasize intra-individual process at the individual level, this idiographic approach aims to unravel the interplay of genes and environments at a more microscopic level-at the individual level and at the micro time scale (e.g., daily). After all, developmental change does not happen overnight but occurs under the continuous coaction of genes and environment in real time at every month, week, day, hour, and second in real daily life contexts. This novel approach poses new questions to the theory of behavioural genetics in intra-individual developmental processes, new challenges to study designs, data collection methods, and analytic techniques in behavioural genetic research, as well as new opportunities to disentangle genetic and environmental influences in human development at a new and exciting level of analysis. It is our ultimate intention, by introducing this novel idiographic behavioural genetic approach to intra-individual variability, together with an analytic method among others suitable for this type of data, to raise awareness of this approach to more behavioural genetic researchers. We envision and are optimistic that the field of behavioural genetics will offer a lot to our understanding of the interplay of genes and environment in intraindividual variability.

Acknowledgments The authors gratefully acknowledge the ongoing contribution of the participants in the Twins Early Development Study (TEDS) and their families. TEDS is supported by a programme grant from the UK Medical Research Council [G0901245; and previously G0500079], with additional support from the US National Institutes of Health [HD044454; HD059215].

\section{Compliance with Ethical Standards}

Conflict of Interest Yao Zheng, Peter C. M. Molenaar, Rosalind Arden, Kathryn Asbury, and David M. Almeida declare that they have no conflict of interest.

Human and Animal Rights and Informed Consent All families provided informed consent and understood that they were free to withdraw from the data collection at any time. None chose to do so. All procedures were approved by, and performed in accordance with the Institutional Review Board at King's College London.

Open Access This article is distributed under the terms of the Creative Commons Attribution 4.0 International License (http://crea tivecommons.org/licenses/by/4.0/), which permits unrestricted use, distribution, and reproduction in any medium, provided you give appropriate credit to the original author(s) and the source, provide a 
link to the Creative Commons license, and indicate if changes were made.

\section{References}

Asbury K, Plomin R (2013) G is for genes: the impact of genetics on education and achievement. Wiley, Oxford

Asbury K, Almeida D, Hibel J, Harlaar N, Plomin R (2008) Clones in the classroom: a daily diary study of the nonshared environmental relationships between monozygotic twin differences in school experience and achievement. Twin Res Hum Genet 11:586-595

Baker LA, Cesa IL, Gatz M, Mellins C (1992) Genetic and environmental influences on positive and negative affect: support for a two-factor theory. Psychol Aging 7:158-163

Bell JT, Saffery R (2012) The value of twins in epigenetic epidemiology. Int J Epidemiol 41:140-150

Bell JT, Spector TD (2011) A twin approach to unraveling epigenetics. Trends Genet 27:116-125

Birkhoff GD (1931) Proof of the ergodic theorem. Proc Natl Acad Sci 17:656-660

Boker S, Horn E, Meyer M, Turkheimer E (2014) A method for ACE decomposition of parameters of dynamical systems [abstract]. Behav Genet 44:649

Boomsma DI, Molenaar PCM (1987) The genetic analysis of repeated measures I: simplex models. Behav Genet 17:111-123

Burt SA, Klahr AM, Klump KL (2015) Do non-shared environmental influences persist over time? An examination of days and minutes. Behav Genet 45:24-34

Chow S-M, Ram N, Boker SM, Fujita F, Clore G (2005) Emotion as a thermostat: representing emotion regulating using a damped oscillator model. Emotion 5:208-225

Clark LA, Watson D (1988) Mood and the mundane: relations between daily life events and self-reported mood. J Pers Soc Psychol 54:296-308

Cleveland HH, Almeida DM (2013) A behavioral genetic investigation of weekend drinking among an adult sample. Subst Use Misuse 48:359-370

Collins F (2010) The language of life: DNA and the revolution in personalized medicine. HarperCollins Publishers, New York

Diehl M, Hooker K, Sliwinski MJ (eds) (2015) Handbook of intraindividual variability across the life span. Routledge, New York

Dolan CV (2005) MKFM6: multi-group, multi-subject stationary time series modeling based on the Kalman filter. Available to be downloaded for free from http://quantdev.ssri.psu.edu/resources

Dolan CV, Nivard MG, van Dongen J, van der Sluis S, Boomsma DI (2015) Methylation as an epigenetic source of random genetic effects in the classical twin design. Adv Genomics Genet 5:1-11

Eaves LJ, Silberg JL, Hewitt JK, Rutter M, Meyer JM, Neale MC, Pickles A (1993) Analyzing twin resemblance in multisymptom data: genetic applications of a latent class model for symptoms of conduct disorder in juvenile boys. Behav Genet 23:5-19

Eid M, Diener E (1999) Intraindividual variability in affect: reliability, validity, and personality correlates. J Pers Soc Psychol 76:662-676

Ferrer E, Nesselroade JR (2003) Modeling affective processes in dyadic relations via dynamic factor analysis. Emotion 3:344-360

Fraga MF, Ballestar E, Paz MF, Ropero S, Setien F, Ballestar ML, Boix-Chornet M (2005) Epigenetic differences arise during the lifetime of monozygotic twins. Proc Natl Acad Sci USA 102:10604-10609

Gatz M, Pedersen N, Plomin R, Nesselroade JR, McClearn GE (1992) Importance of shared genes and shared environments for symptoms of depression in older adults. J Abnorm Psychol 101:701-708
Gillespie NA, Neale MC (2006) A finite mixture model for genotype and environment interactions: detecting latent population heterogeneity. Twin Res Hum Genet 9:412-423

Harvey AC (1989) Forecasting, structural time series models and the Kalman filter. Cambridge University Press, Cambridge

Haworth CMA, Davis OSP, Plomin R (2013) Twins Early Development Study (TEDS): a genetically sensitive investigation of cognitive and behavioral development from childhood to young adulthood. Twin Res Hum Genet 16:117-125

Hunter M, Bard D, Beasley W, Meredith K, Rodgers J (2014) A dynamic mixture biometric model of cognitive development in the NLSY children [abstract]. Behav Genet 44:663

Jacobs N, Rijsdijk F, Derom C, Vlietinck R, Delespaul P, van Os J, Myin-Germeys I (2006) Genes making one feel blue in the flow of daily life: a momentary assessment study of gene-stress interaction. Psychosom Med 68:201-206

Jacobs N, Menne-Lothmann C, Derom C, Thiery E, van Os J, Wichers M (2013) Deconstructing the familiality of variability in momentary negative and positive affect. Acta Psychiatr Scand 127:318-327

Kan K-J, Ploeger A, Raijmakers MEJ, Dolan CV, van der Maas HLJ (2010) Nonlinear epigenetic variance: review and simulations. Dev Sci 13:11-27

Kelderman H, Molenaar PC (2007) The effect of individual differences in factor loadings on the standard factor model. Multivar Behav Res 42:435-456

Kessler RC, Gilman SE, Thornton LM, Kendler KS (2004) Health, wellbeing, and social responsibility in the MIDUS twin and sibling subsamples. In: Brim OG, Ryff CD, Kessler RC (eds) How healthy are we? A national study of wellbeing at midlife. University of Chicago Press, Chicago, pp 124-152

Klump KL, Keel PK, Racine SE, Burt SA, Neale M, Sisk CL, Hu JY (2013) The interactive effects of estrogen and progesterone on changes in emotional eating across the menstrual cycle. J Abnorm Psychol 122:131-137

Loehlin JC (1996) The Cholesky approach: a cautionary note. Behav Genet 26:65-69

Martin NG, Eaves LJ (1977) The genetic analysis of covariance structures. Heredity 38:79-93

Menne-Lothmann C, Jacobs N, Derom C, Thiery E, van Os J, Wichers M (2012) Genetic and environmental causes of individual differences in daily life positive affect and reward experience and its overlap with stress-sensitivity. Behav Genet 42:778-786

Molenaar PCM (1985) A dynamic factor model for the analysis of multivariate time series. Psychometrika 50:181-202

Molenaar PCM (2004) A manifesto on psychology as idiographic science: bringing the person back into scientific psychology, this time forever. Measurement 2:201-218

Molenaar PCM (2007) On the implications of the classical ergodic theorems: analysis of developmental processes has to focus on intra-individual variation. Dev Psychobiol 50:60-69

Molenaar PCM (2010a) On the limits of standard quantitative genetic modeling of inter-individual variation: extensions, ergodic conditions and a new genetic factor model of intra-individual variation. In: Hood KE, Halpern CT, Greenberg G, Lerner RM (eds) Handbook of developmental science, behavior, and genetics. Blackwell, Malden, pp 626-648

Molenaar PCM (2010b) Testing all six person-oriented principles in dynamic factor analysis. Dev Psychopathol 22:255-259

Molenaar PCM (2014) Dynamic models of biological pattern formation have surprising implications for understanding the epigenetics of development. Res Hum Dev 11:50-62

Molenaar PCM, Boomsma DI (1987) The genetic analysis of repeated measures II: the Karhunen-Loeve expansion. Behav Genet $17: 229-242$ 
Molenaar PCM, Campbell CG (2009) The new person-specific paradigm in psychology. Curr Dir Psychol Sci 18:112-117

Molenaar PCM, Raijmakers MEJ (1999) Additional aspects of third source variation for the genetic analysis of human development and behavior. Twin Res 2:49-52

Molenaar PCM, Boomsma DI, Dolan CV (1993) A third source of developmental difference. Behav Genet 23:519-524

Molenaar PCM, Smit DJA, Boomsma DI, Nesselroade JR (2012) Estimation of subject-specific heritabilities from intra-individual variation: iFACE. Twin Res Hum Genet 15:393-400

Muthén B, Asparouhov T, Rebollo I (2006) Advances in behavioral genetics modeling using Mplus: applications of factor mixture modeling to twin data. Twin Res Hum Genet 9:313-324

Neale MC (2014) Latent classiness and other mixtures. Behav Genet 44:205-211

Neiss M, Almeida DM (2004) Age differences in the heritability of mean and intraindividual variation of psychological distress. Gerontology 50:22-27

Nesselroade JR (1991) The warp and woof of the developmental fabric. In: Downs R, Liben L, Palermo D (eds) Visions of development, the environment, and aesthetics: the legacy of Joachim F. Wohlwill. Erlbaum, Hillsdale, pp 213-240

Nesselroade JR, Molenaar PCM (2010) Analyzing intra-person variation: hybridizing the ACE model with P-technique factor analysis and the idiographic filter. Behav Genet 40:776-783

Nesselroade JR, Ram N (2004) Studying intraindividual variability: what we have learned that will help us understand lives in context. Res Hum Dev 1:9-29

Nesselroade JR, Gerstorf D, Hardy SA, Ram N (2007) Idiographic filters for psychological constructs. Measurement 5:217-235

Petronis A (2010) Epigenetics as a unifying principle in the aetiology of complex traits and diseases. Nature 465:721-727
Plomin R (2011) Commentary: why are children in the same family so different? Non-shared environment three decades later. Int $\mathbf{J}$ Epidemiol 40:582-592

Plomin R, Daniels D (1987) Why are children in the same family so different from one another. Behav Brain Sci 10:1-16

Ram N, Gerstorf D (2009) Time-structured and net intraindividual variability: tools for examining the development of dynamic characteristics and processes. Psychol Aging 24:778-791

Riemann R, Angleitner A, Borkenau P, Eid M (1998) Genetic and environmental sources of consistency and variability in positive and negative mood. Eur J Pers 12:345-364

Shiffman S, Stone AA, Hufford MR (2008) Ecological momentary assessment. Annu Rev Clin Psychol 4:1-32

Stone AA, Shiffman S, Atienza AA, Nebeling L (2007) The science of real-time data capture: self-reports in health research. Oxford University Press, New York

Walls TA, Schafer JL (eds) (2006) Models for intensive longitudinal data. Oxford University Press

Watson W (1988) Intraindividual and interindividual analyses of positive and negative affect: their relation to health complaints, perceived stress, and daily activities. J Pers Soc Psychol 54:1020-1030

Wichers M, Myin-Germeys I, Jacobs N, Peeters F, Kenis G, Derom C, van Os J (2007) Genetic risk of depression and stress-induced negative affect in daily life. Br J Psychiatry 191:218-223

Wright FA, Sullivan PF, Brooks AI, Zou F, Sun W, Xia K, Abdellaoui A (2014) Heritability and genomics of gene expression in peripheral blood. Nat Genet 46:430-437

Zevon MA, Tellegen A (1982) The structure of mood change: an idiographic/nomothetic analysis. J Pers Soc Psychol 43:111-122 